Conclusion Inpatient rehabilitation for patients with Long Covid was associated with improvement in exercise performance, lung function and psychological parameters with and without hospitalization during the acute COVID-19 infection. Results indicate the usefulness of rehabilitation to reduce and avoid long-term consequences of a COVID-19 infection independent of the severity of the acute course of the disease.

\section{P72 USING THE MULTIDIMENSIONAL DYSPNOEA PROFILE IN COVID-19- THE DIFFERENT SENSATIONS OF BREATHLESSNESS AND THEIR IMPACT}

${ }^{1} \mathrm{E}$ Daynes, ${ }^{1} \mathrm{C}$ Gerlis, 'L Houchen-Wolloff, ${ }^{1} \mathrm{~N}$ Gardiner, ${ }^{2} \mathrm{SJ}$ Singh. ${ }^{1}$ Centre of Exercise and Rehabilitation Sciences, NIHR Leicester Biomedical Research Centre-Respiratory, Leicester, UK; ${ }^{2}$ Department of Respiratory Sciences, University of Leicester, Leicester, UK

10.1136/thorax-2021-BTSabstracts. 182

Introduction Patients with COVID-19 can experience breathlessness during the acute phase of the illness and as a longterm symptom following infection. Breathlessness can be a distressing symptom that manifests as a number of different sensations. The Multidimensional Dyspnoea Profile (MDP) explores different sensations of breathlessness and the emotional impact of these sensations. The aim of this study is to understand the prevalence/severity and sensations of breathlessness following COVID-19.

Methods Patients with COVID-19 who were discharged from the University Hospitals of Leicester between March 2020 and November 2020 were called as part of routine clinical follow up and invited to take part in this research. The MDP was administered over the phone by a clinician. Data was analysed in SPSS, using an independent t-test. The MDP is presented as immediate perception and emotional response (scored out of 60 and 50 respectively, higher $=$ more severe).

Results 280 patients (mean [SD] age 57[13] years, gender $\mathrm{n}=161 \quad(56 \%)$ male, $\mathrm{n}=155(54 \%)$ white British, 80(29\%) with a pre-existing respiratory condition) completed the assessment. The mean [SD] length of stay was $10[15]$ days, time to follow up 47[31] days, and 25 (9\%) of patients were ventilated. The mean [SD] of the MDP was 13[15] for the immediate perception and 9[11] for the emotional response (figure 1).

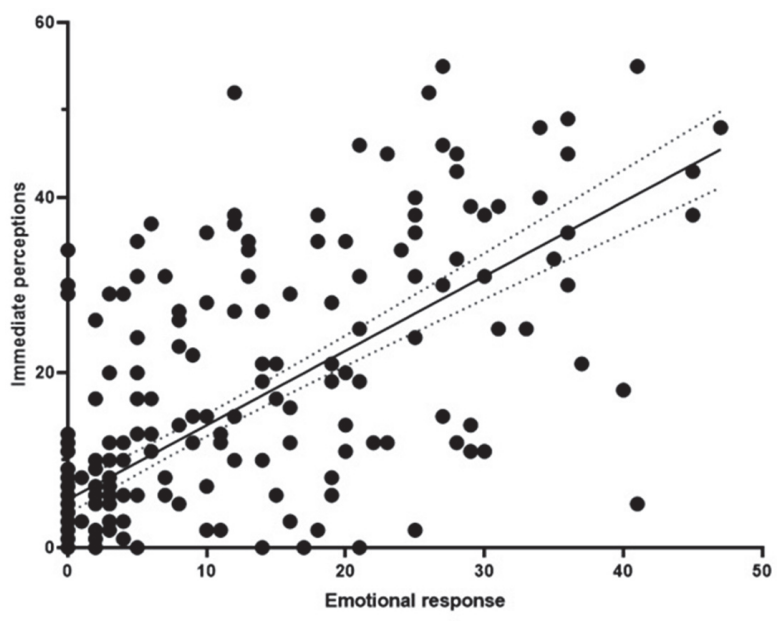

Abstract P72 Figure 1 Immediate and emotional domains of the Multidimensional Dyspnoea Profile
Of those reporting breathlessness $(177(63 \%))$ mean [SD] of $20[15]$ in the immediate perception and 13 [12] for the emotional domain. The most prevalent sensation was hyperventilation and, emotion was frustration. There were no statistically significant differences between the mean [SD] of the immediate response or emotional domain between ventilation status, and length of stay. Females reported a statistically significantly higher immediate and emotional response than males (mean [SD] difference 7[2], $\mathrm{p}=<0.01 ; 6[2], \mathrm{p}<0.01$ respectively). There were no significant differences in the immediate or emotional domains in those with or without a pre-existing respiratory condition $(\mathrm{p}=0.25)$.

Conclusion $63 \%$ of patients following COVID-19 identified at least one sensation of breathlessness that persisted after discharge. The severity and emotional response to breathlessness was not influenced by length of stay, ventilatory status during admission or pre-existing respiratory condition.

\section{Virtually perfect: remote medicine and digital health}

\begin{tabular}{l|l}
\hline P73 IMPLEMENTATION OF A COMPUTER GUIDED \\
CONSULTATION (INTELLIGENT CLINICAL DECISION \\
SUPPORT SYSTEM SOFTWARE) IN THE LIVERPOOL \\
SLEEP SERVICE: THE CREATION OF A DIGITAL \\
ECOSYSTEM TO TRANSFORM PATIENT PATHWAYS
\end{tabular}

${ }^{1} \mathrm{~B}$ Chakrabarti, ${ }^{1} \mathrm{RM}$ Angus, ${ }^{2} \mathrm{P}$ England, ${ }^{1} \mathrm{M}$ Ahmad, ${ }^{1} \mathrm{M}$ Brady, ${ }^{2} \mathrm{MG}$ Pearson, ${ }^{2} \mathrm{E}$ McKnight, ${ }^{2} \mathrm{~L}$ Reed, ${ }^{1} \mathrm{M}$ Thomas, ${ }^{1} \mathrm{SE}$ Craig. ' 'Liverpool University Hospitals NHS Foundation Trust, Liverpool, UK; ${ }^{2}$ Lunghealth Ltd, Swaffham, UK

\subsection{6/thorax-2021-BTSabstracts. 183}

Background The Liverpool Sleep Centre provides secondary/ tertiary level care for a range of sleep disorders. An increasing volume of referrals and the impact of Covid threatened to overwhelm the service resulting in increasing clinical risk and decreased patient satisfaction. We describe how the use of technology addressed these challenges through the implementation of a Computer Guided Consultation system i.e. clinical decision support software (CDSS).

Methodology The CDSS is a digital ecosystem comprising multiple intelligent consultations encompassing the entire OSA pathway including Assessment and diagnosis, CPAP set up, CPAP monitoring and issuing consumables thus acting as an end to end system solution and an Electronic Patient Record. The CDSS also features a 'clinical dashboard' allowing the service to track activity, monitor RTT performance and identify high risk patients e.g. sleepy drivers, hypoventilation in 'real time'. Results Prior to implementation of the CDSS, all suspected OSA referrals underwent a sleep study and the results of which together with the information contained in the referral letter would have been reviewed by a Consultant in a 'Virtual clinic' with treatment decisions made in such clinics. In order to meet this demand, the service required 8 clinics weekly (5 Consultant 'Virtual' clinics consisting of 20 patients each and 3 'Combined' Consultant/Physiologist clinics). Since March 1st to June 2021 following CDSS implementation, 325 patients (see table 1 for demographics) with suspected OSA were assessed by paramedical staff using the CDSS. Only $15 \%$ of these patients subsequently required a Consultant review either in a 'Virtual' or a 'Face to Face' manner (translating into just 0.5 clinics weekly), no 'combined' clinics were required with 